\title{
Bejelgesverzetidnis.
}

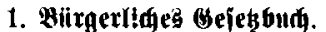

\$12 Firmenrecht 5.282.

29 \&ojhung bes eingetragenen (Heidäfts. fiifrers 204 R. 1.

37 Beruץung ber Berjammlung 197.

59 Y3rüfung, oh der wirffiche Borjtand an. gemeldet hat 206

107 shebeutung 90.

117 Beridjleierte Sadjgriutoung 304.

119 gefürbte Rahrungsmittel 69 .

138 Bertrag ïber Nidhtangabe Des Sdyuäl. gerers 91 .

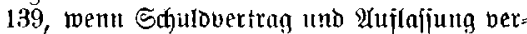
eutigt jutlo 219 .

141 bei Puflaffung (Hejichäft

164 bie bom Bornumb exteilte Bollmadt 184.

167 unvitsernfflide Bollmad)! zu Brmulo-

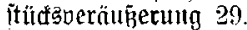

170 Machweis des Fortbeitehens 66.

177 bei genehmigungasbedürftigen bejouäịten 211,213 .

181, wem ber lisertreter oic 3uttinmung bor Bertretenem bem Bertragageguer erłärt 358 .

185 seilung 1 ; bei \$wijdenerwerbern 176 .

242 vorbehaltote QQiejerungšmöglidgfeit 19 ; alterl'ntive Crmäditịnng 23 ; Nadhählen ber Belojideinpoìd)en 39 .

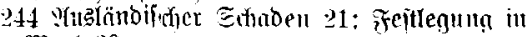
Mart 20.

$\because 49$ Edadenberedmuna bei nidtigem Def:

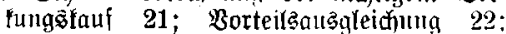
Berzidat auf Eráą 23.

262 :llternative Ermäbtigung 23.

$2673 \mathfrak{a}$ hlung einea Drittest 24.

269 hei Banffdulden 25; für 9lızahlung anti den Taufpreiz $26 \mathfrak{R} .1$; "ein vom (Släubiger anzugebender $5 \mathrm{rt}^{\prime \prime} 26$.

-274 gegenilber $\$ 1042$ ЗßS. 105 : nenn beine Teile $\mathfrak{A}$ trprühe habell 106.

276 verfehrserforderlifje Sorgialt 26,$27 ;$ un berficherte łtberfenoumg einer verpiänoeten this 18.

288 Balutajhaden 103/5.

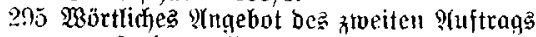
wegen früherer Mängelrüae 28 .

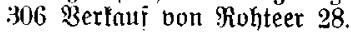

313 23ertrag über Einfringuing boil Brums.

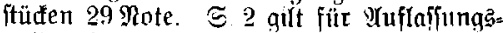
vollmaditen 30,65

315 Berveis̆aft 31

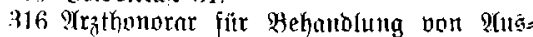
fäubern 31; Berqütuma ber Şdiebsrihter 169 ․ 1.

126 bei teilmeijer Seiitung 32, 106; Ufbuahnte.

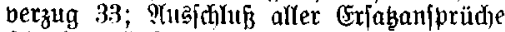
34; Der Jaftung bei reinmaichinen 46; Sufzelfublieferung 35; Seben einer Ittber= Yegungäfrift 36; nach angeoroneter Beichäits. alffïht 37; wenn Beferlfafafter 2tuflölung uno (Sefabôftsäbergantg bereinhoren 291 .

361 Meihnahtölieferung 38 .

368 3orbehalt 40 .
\$ 410 Inzeige bes $2:$ :bi. S. 41.

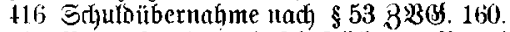

13:3 Berfäufer, ber virtífoftlich nur Bermitt ler iit 41 .

434 beidlaguabme 42 .

437 beim Sundel init Sd)ed' auf Wien $4 \geq$.

44 bei Araftrages 43.

116 Bejahr ber $\mathfrak{B e j}$ )lagnahme 44 ; bei ult ridtiger Berjelioung 44.

147 Berjentungsart 45; Berjidjerung 45 .

459 8uficheruitg 46.

477 औ65. 2 Cribe Der lliterbrechung 47.

480 Erfüillung 8 ugunzug 69 N. 1 .

504 Rüdwirfung bes gejeblidien 2 orfant

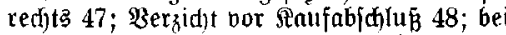
jormtwiorigent Rertrage 49; bei Srunt

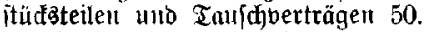

j10 Mitteifung nad 1 âf. 48.

516 Begriff ber Sdgentung 52, ourd) preimilli= gen lunterhalt $5 \mathbf{3}$.

o31 Redtstolgen 156

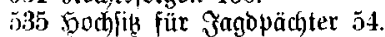

336 Dientebung und Ginftellung ber Sanmel. heizung 247 .

549 saftung fïr Rachmieter 70 .

ว558 Beieitigung von (Sebäubereften 55 .

559 bei nadhträglicher Buitimmung bes $\mathfrak{W}_{0}$ h. itungsamts 55 .

611 "PFleqevertrag" 70; Sఝ̆aupieler 70 $\mathfrak{R . 1 a ; ~ L e f r l i n g ~} 70$ '. 2 b; $\mathfrak{B}$ erfilmung 70 R. $2 \mathrm{c}$.

(18 renfterpubeu 73; Qebrerimmer 74.

626 Urbeitsverweigerung 114.

631 Rabatte" in Q̂nzeigengewerbe 76.

638 :́rbeiten bei Baumerfen 76.

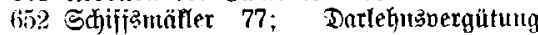
$70 \Re .1$

674/5 ?Irfprud des Geridhtabollziegers auf Erịtattung ber $\mathfrak{U}$ miabíteller $78 \mathfrak{R} .1$.

675 Gaftung ber Banf gegen ihren 2Huftraggeber 78.

676 YUäfunft über Dectung bon Sd)edß 79. 685 crrftattutigbabficht 78 R. 1.

687 bei ßfänbung eigener Săhen des Bläu. bigers 155 .

688 gegenüber bem Saftwirt 82,116 ; RTetber ablage bes Theaters 115; Ginf(b)ränfutna ber Softung 115

701 ङ eingebradte Fahrzeuge 81 ; Begriff bes Begleiters 81; Berwahrung ber Bimmer. idiüffel 83.

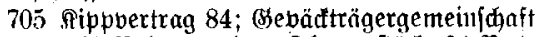
84; Vhtffïhrung eines Theateritü $\mathfrak{F}_{3} 84 \mathfrak{R}$. 1 .

741 Ređjtşgemeinjdyaft am Befib 208.

743 Mitbenubung einer Leiter 5 .

748 Eintragung ber interhaltäpflid)t 5 .

759 "Pflegevertrag" 70.

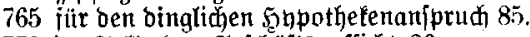

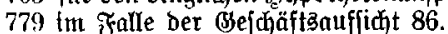

783 "Liefexjđein" 86; Mflrebitio 88 u. $\mathfrak{R} .1$.

807 "Sutjhein 88. 
$\$ 812$ qneigmung bejdlagnahmter Sađjen S. 89. 814 bei Bahlung eines Dritten 24.

816 "Berfügung" 90.

817 Bertrag über Nichtangabe des 厄dimän= gerers 91 .

$\$ 18$ "nid)t mehr bereidert" 90.

823 Streif 92,101 ; Cinfriebigungen 92: glatte Treppe 93.

826 Einflagung von Prozekfoiten 96; Reh-

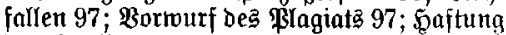

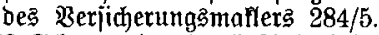

833 Sdjeuwerben burd) Alnfurbeln 94; sunbe länolicher Unmejen 98

839 Bertreter bes Rotars 98; ßoptbeamte 99;

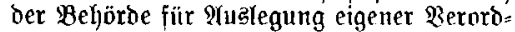
nungen 100.

854 Mitbefí an Mieträumen 208.

854 Befibjđu bei (Enteignungen 208; (cha= dengerias megent sigenmadi 209.

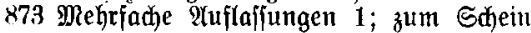
173; (Se đajftsunfähiger 210 ; an bie Santols: vertretung ber Eowiet=Republil 210 .

874 Ungabe ber Şöhe ber Belbrente 10.

877 Bebeutung 2 ; lumbanblung in mert= beitänbige Sypothefen 217.

880 nidit antwenbbar auf gefeblidie shang= vorkehalte $3 \mathfrak{R}$. 1 a.

881 Bebeutung $4 \Re, 1$ b

892 bei Enteignung 4 .

905 Brefähroung shaft 218.

925 Bereinigung bon छrfulboertrag Into IYuflaffung 219.

929 bei laberjenoung uno Burberfïgung= ftellung 220, 222; hei (5rmerb bon Miteigen= tutm 222.

950 Berfinneiben bon Bilogruppen $223 \mathfrak{\Re} 1$.

1004 gegen öffentliche Sportpläbe 223.

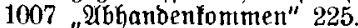

1010 Cintragung ber lunterhaltspflicht 5 .

1018 Belaftung zugunften reeller Brumbitïđas: teile 6 .

1025 "Teilung" 6

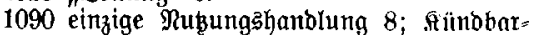
feit, Unterhartapflidyt 225.

1093 mietähriliỏe $\mathfrak{B e f t i m m u n g e n ~} 8,225 \mathfrak{N} .1$

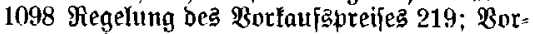

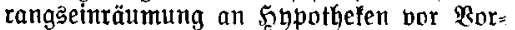
fautsisechten 225 .

1103 gilt aud für perfönliche Diențtbarfeiten 2.

1105 Reallajten 226; beftinmter ऊribalt 9;

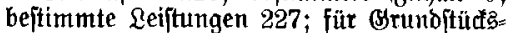
anteile 6, zur Exrinttung eines Zaunz $9 \mathfrak{N}$. 1; Yufwertung 226; bon Unterhaltabeiträgen 11; Bahlung in Bold 11.

1115 3infegzinjen wertbeftänbiger $\mathfrak{B f a n o b r i e f =}$ barlehen 229; Shpothefen it Feingold 232.

1190 Beftellung bon Teilhypothelet 14,16 ; mertbeftänoige Gödithnpothef ohne frcit= jebuntg eines Stidjtages 233.

1199 Rentenjwuld auj Bett 17.

1223 Erfülltunģ̄ort 18.

$1304^{2}$ bei entmintigten SSeifteş4hwachen 347

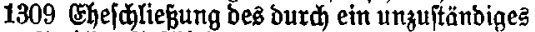
Geriajt Gefátebetten 347 .

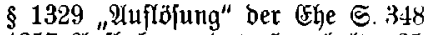

1357 शufhebung bes Soußhalte 351/2.

1358 fiütoigungäred)t bes Mannes 352

1361 Beftimmtheit ber Belorente 353; aujer. orbentridye $\mathfrak{A} u s g a b e n$ I54

1373 Mitbefíb bes Mamues $354,3559.1$

1402 Bezeiđnung bes Redtsgejđäfts 356 .

1405 Bebeutung 356.

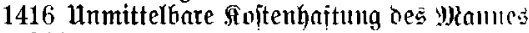
355.

1439 Begrifi des Eottoerguts 356 .

1445,1487 Sintoilligung ber ?(btömm!iuge $35 \times$.

1502 Bebentung 360

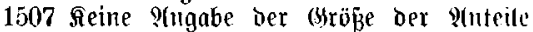
$361 \Re .1$

1549 Redht bes überlebenden 361 .

$1631^{2}$ zur Durd)fübrung eines Puientbults wedffels 366; religiöje (5rziehumg 367 .

1636 Berlehrs̈regelung $\mathbf{3 8 6}$.

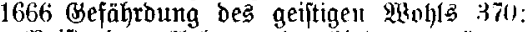

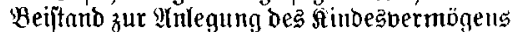

371 ; Sdubauffid)t 371 গ. 1.

1677 Epäterer $\Re$ Segiall 369 .

1685 Fiirforgeerziehung gegell die \$lutter $\$ 369$.

1717 Redeutung bes 2. 96f. 373.

$1728^{2}$ ohne vorherige benehmigung $375 \mathfrak{R} .1$.

1750 Bertragşนhlu 374 .

1773 Bormunobeftellung ohue oic gejeglidgen

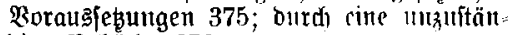
bige Behörbe 376 .

1778 "ïbergangen" $377 \Re .1$.

1779 Religion bes Beruү̧טormunds 377.

$1793 \mathrm{im}$ Falle ber Firiorgeer3icbung 379.

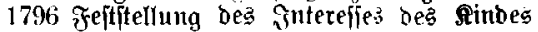
365 .

1804 Watm entspridt ein Bertan bem Müll= belintereffe? 380,382 .

1812, went ber bom Bormuno Reovllmät) tigte nad) ber Bolljährigheit oes Mlïubers Die $\mathfrak{Q}$ bffung berwilligt $\mathbf{1 8 4}$

1820 Bebeutung bes 3. Yb 18.1

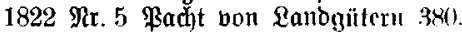

1829 Doppelbollmad)t zur (Entgraerutubutc und Mitteilung ber (Senel)mign!g 382 ; Sher zicht auf diefe Mitteilung 384 .

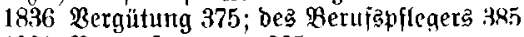
1906 Roraujebungen 385 .

1909 厄)ubauffitit 385.

$1951^{2}$ "aus demfelben (s)runto" 386.

1960/1" Ungetwißheit Der Prutnhunc 388; bs Racherbent 389

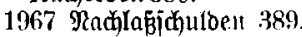

2027/8 2usktutfterteiltug 389 .

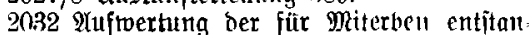
Denten Shpothefen 390 .

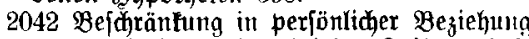
392; Schreibberjehen bei ber Teilutg 393 2065 crbeinjebung unter her sebingung hes Bohlberhaltens 394 .

2094 bei nidtiger Grbeinfebuth 394.

2096 „22egfall" 394.

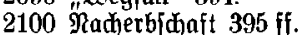

2102 Bebeutung bes 2. 2 (b). 396.

$2108^{\top}$ Bebeutung 399. 
$\$ 2113$ Borbehaltlofe Sutttung über 5 ypothefen 5. 175; Binterhbhung bes porerben 191 . 2124 abweidente Bereinbarung 400 R. 1 . 2136 Tie Racherbfolge fanm an eines bou mehreren (creignifien gefniipit jein und im rebterent Fall ben Borerben ein Berutädhtnię zugetendet merben $4(00$; Bebingung, tan ber Rorerbe nidjt antoers verï̈ght 404 .

2197 Anorbutung 400, $401 \mathfrak{R} .1$.

2200 (Expudit $401 \Re$.

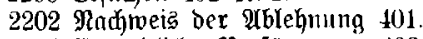

2205 (Entgeltliduc Berfügungen 403

2215 ift berzid)tbar 403 .

2269 Bebeuhung 185 , Sadyerbidhait nòn $\mathfrak{N i e \tilde { B }}$ braudhverntä()tıie 397.

2325 Bebeutung 287.

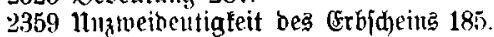

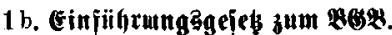

136 Bebelltulig bex $\mathfrak{R r} .1377$.

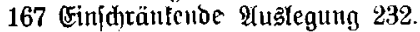

$208^{1}$ Solbjas 2 wirft nidyt zutü̈f 364 .

\section{2. ตanbelägeieğbud.}

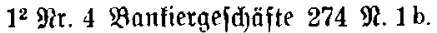

2 nidit mehr beftehenoe Bsejellidiaften 275.

8 Qlufnahme ita Regifter 274 $\mathfrak{R} 1$

12 Cinreidung ber finmelsungen 274 ; formlos wiberruftich 204; Burïdnahme bes 2 giderrıfङ 299.

15 Eduus des Dritter 276.

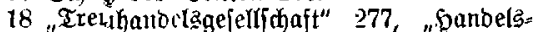

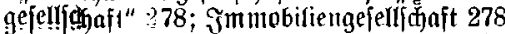
$\mathfrak{R} .1 \mathrm{a} ; \quad$ (bllibeherd $=$ Bertrieb $278 \mathfrak{R} 1 \mathrm{~b}$; 5.er YItstiquariat $278 \Re .1 \mathrm{c}$; Frmmentum 278 R. 1d: Lallowirtidaftlidje Budjptelle

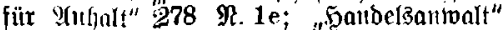

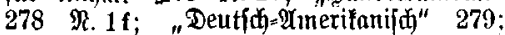
Mittelbeutidue "Tertilgef. 280; "fïr bell idflefilchen श[ocl" 280.

19 Rame bes Fommandititen 280.

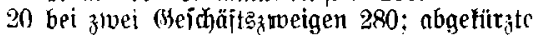
frima 280 .

22 abgeleitetc Firıta, fpätere Sintorntuta 280.

29 5anbelaniederlajiung 280.

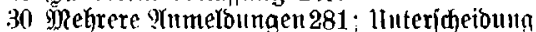
281 अi. 1.

37 Bebentung bos 2. 96f. 282.

:0 bei eincr nad \& $232^{2}$ geregelten Rorftallos vertretıng 282: Rejd)rönfuma แnd शु. . $283 \Re .1$.

54 Berveis̆laft für abichlïife 283 .

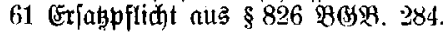

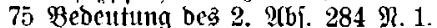

89 "für" uno "aus" bem

93 siftung bes Berjiderungsmaflers 285.

120 Reingeroin bei Belbentwertung 286: (servimunteile, bie bes Crben zu sahlen iiito 287

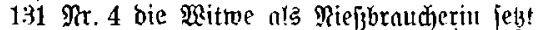
die Sejelljwaft nidht fort $289 \Re$.

139 cintritt eines miterben 289 尺. 1
$\$ 142$ Urteil auf 2 fuftöung $\subsetneq .289 \mathfrak{R . 1}$; 2lbrede, bas bas Grumbitüt erit und gezahlter $\mathfrak{x}$ b: finbung iibergeht 289; Nadjweia bez über= gangs ber Sinpotheten 289 .

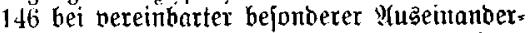

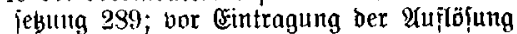
290 ; nad) ber

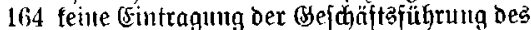
siommantoitifiten $274 \mathfrak{R} .1$.

$182 \Subset .1$ fceilung bes Mangels292; leinte :Rüt = wirtung ber Solbbilanj?BS. 294/5.

$183 \mathrm{umwandung}$ bon Ramen aftien 298

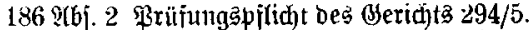

187 Qnmentung auf bis Siapitalserhohung 299.

$188^{1}$ Die ftbernahme ift entficibeno, fpätere Fbänderung unerheb!ich 296 ; $\hat{\text { trapitals }}$ erhöfung uvd) vor (Fintragung ber $\mathscr{Y}(\mathrm{S} .299$.

191 if., wenu bor ber (Eintragung ber $\Re(S$. bas Supital erhäht unb bon beu Grintibern über Jummen wiro 300 .

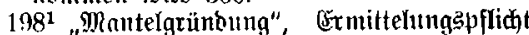
296.

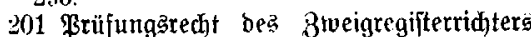
$292,301,318$.

207 Berichleterte Endigrinoung, bie erit burdh eine gemäß $\$ 207$ borgennmmete Sad)= griimbunt erfennbar wirb 303, 306; Crin= tragung einter fFirmenänderling $305 ; \mathfrak{B e}$. beutung bez $\$ 207^{3}$ 307; ijt auth bie $\mathfrak{2}$ f laffung untwirkfan? 307 .

211 ff. Tuferlegung ven bilfäpfliḑ)ten bei

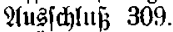

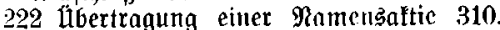

$232^{2}$ Yedeutung des 2. Sabes 301

243 Rerpflidtung zur $\mathfrak{W a h l}$ einer $\mathfrak{2}$ erion in ben $\mathscr{A} u f f i d t$ tsent 311.

246 Teilnahmered bes Yufintstats an ben Beneralverfamm! !ntgen 311: die Gabutge

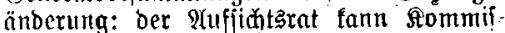
fionen zur 2ubübung feiter Rejugniffe or: nement $311 \mathfrak{R} .1$.

254 Ontoenbung bes $\$ 256$ 31:; Rebeutnun 그 $\$ 254^{3} 5 \mathrm{HB} .313$

255, 256, 259 Aeine Beurfunbuta des $\mathfrak{B e r}$ zidhts bei uniberfalberjammlutgent 314 .

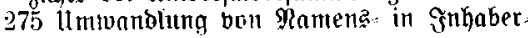
altien 398.

277 (Sejebrviorige (5rhöhungăbejhlülfe 316: nod) in Yapiermuart $\mathbf{3 1 7}$.

-278 Bebentung des. 3. 2tb. 316 .

281 bei her lubernahme beg erhöhten Stapitale unantwend bar 300: unberbintilith geworbente Zeidynungen 316 .

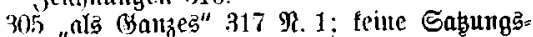

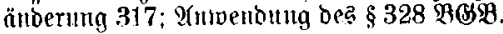
$317 \mathfrak{R} .1$

306 Entfprechentoc ofutwentuma bes $\$ 304^{\circ}$ () $13.319 \Re .1$

309 Bedeutung 296; bei beridfleierter Ead) griinoung 304

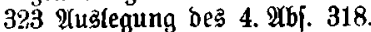

340 IItseinanberjegung 319. 
3. Gejek betr. Die Gejerlidiajten mit be: jüräntter saftung.

$\$ 3$ Bebeutung ber $\mathfrak{N x} 4$ 5. 324.

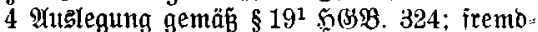
ipradilide Bezeidnung 324

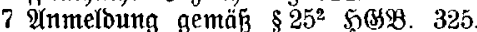

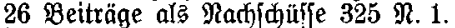

$35^{2}$ Die Bertretung fann nur int Bejerlfchaftas. vertrage geregelt merben 325 .

38 (Enthebung auf STntrag eines (Sejellidafters $325 \Re .1$; went bie Thaswahl neuer Be¡đäftżülhrer bem Regifterridhter überlaffen ift 325 .

b3 lumbenennung ber firma ber 3rweig nieberlajiung 326 .

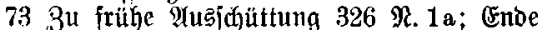

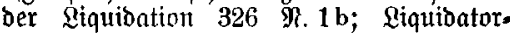
Ređtäantoalt $\mathfrak{R} .1 \mathrm{~b}$

\section{Sejeb betr. bie (Erwerbs= unb 23 irtidhaits:} gentofienidiaten.

22 Bufanmenlegung mehrerer shejdjäftäan. teile in Bolbmart 320 .

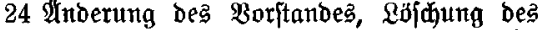
alten 322: Entlajiung on Boritanosmit= gliebern 322; $\mathfrak{B a h l}$ eines Pidhtgenoffen in den Borítanto $323 \Re .1 \mathrm{~b}$.

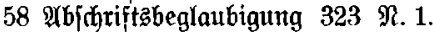

78a \&öfhung ber Ouflöfung zeintragung auf Bejdwerbe bes Reviftonsberbanoş 323.

160 gegen einen zum Boritande gewählten ’idhtgenoffen $323 \mathfrak{R} 1$.

\section{Gejeks gegen uthlnuteren $\mathfrak{B e t t b e w c r b .}$}

1, 3 भusfpiełung von Betwinnen unter ben

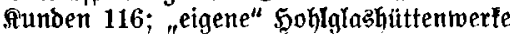
117.

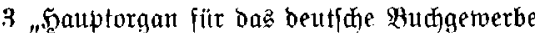
117.

16 Boraugfebungen ber Berrechllungagefahr 118; Shut ber Telegrammanjorift gegen= utber Warenzeidhen 120; Sdus ber Film= bezcichnunger 121.

\section{Pr. Faditidutgorbnung}

v. 27. September 1922.

1, 2 Zuitänoigfeit des ВER. 267/8; bei sin= beziehung von $\mathfrak{W}$ ohntäumen 58; für den Biehgräjungäbertrag 109.

1, 3 ßadit für beıt eigenten Bedarf 269.

2 Seiftunaen, die bie \$adtbeentigung boraus: leber 269; Rarhtragsabfommen 270; Ex=

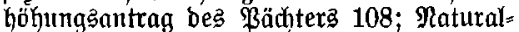
pacht 111; Erhöhung dee porautzuzahlendent Badyt zinjes 59; lebtroillig angeoronete ßact) verlängerung 60 ; vorausjichtlich \{pätere Gelbentwertung 61 ; waln müffen bie $\mathfrak{H} m=$ ftänbe ber Ilnbilligleit borliegen? 63; Ein. flagbare $\mathfrak{Y}$ iniprücthe 111.

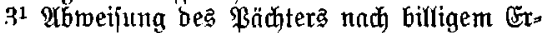
meffen 271; Beredjutttg Des bem ßädfiter verbleibenben Lanbes 272; Befonbere Feft= ftellung ber Notlage 64.
\$5 Berzidgt im Bergleid über bie ßarttoauer ธ. 273.

$12^{2}$ Recht引befhwerde zuläffig 64.

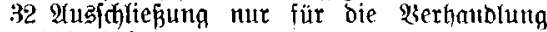
$112,273$.

$31^{2}$ Burüfnahme bes Antrags 106.

\section{Reĭhämietengeies}

v. 24. Măr 1922 .

1 gilt nidht für mitvermietete (Särten 246 .

2 bei geitafieltem Mietzins 334 ; bei 2 burd

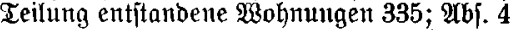
bei vergleidsmeijer Freitfebung ber Friebens: miete 336

4, 5 Ireppenbeleudytung $246 \mathfrak{\Re} .1$; teilmeifed

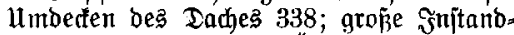
jebungarbeitell in ber inbergangazeit $\mathbf{3 3 6}$.

10 Keich spojt als gerwerblicher Sietrieb 264.

13, 15 Dieniebultg bei sintellung ber ভan= melheizung 247.

22 亿uber bas Borliegen eines "Beidüftge haufe entiheibet bas ME(E). nicht enbaültig 248 ; gefesliche uno preie gliete 266 .

\section{Gejes itber mieterinub unb giegimter.}

$$
\text { v. 1. Эuıi } 1923 .
$$

4 "Berfügungäberedhtigter" 260 .

5 Bollftredung beim Fehlen des säumunga bejehts . 326 .

6 Bejđherbe, wenn ber $\mathfrak{A}$ ntrag erit in ber Berufung geftellt ift 236,329; भHabefferumgs pilint im Falle des 3. 265 . 328 .

7 Reumahl ber Beifiber 237.

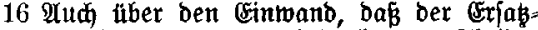
raum in anberer (shemeinbe liegt, entjoibet das M(E). 331.

20 भusjebung tach cab 3 bei Bejhmerbe aus $\$ 6^{2} \quad 329$.

29 aud bei bertraglich betbotener luberlaffung 331.

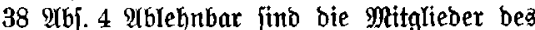
bie (Semeinbe bertretenden Drgans 241.

$40^{5}$ Eirtitiv. UYtoronungen 265 .

$41 \mathfrak{U b}$. 1 Lauf ber Frijt 245.

41 Ub 2 Sab 3 Redt bes Wohnungsిamts 240; gegen 3roangs: mietbertrag 332; feine weitere $B$ ejhroerbe

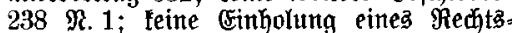
entidieibs bei abroidenden (sntich. auser. preukifdier Befhroerbeltellen 244 .

42 Mbänderunģ̧е ber Bejtwerbeitelle 262.

50 Bollftredung ber bor 1. 10.23 erlaffenen

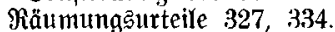

51 Rüưnahme ber vor 1. 10. 23 erteilten (He nehmigung zur Bollftreffung 246,$333 ; 3 \mathrm{u}$.

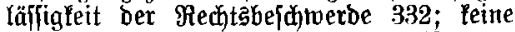
bebingte Benehmiaung nach 2. शYb 333; OG5. 2 gilt aud fiur Bergleidhe, bie bor 1. 10. 23 geristiflic geifloffen find, bor ift bei bem :erfahren bereits bas nete Recht anzutwenden 263. 


\section{Bohnutgakmangetgeicls \\ v. 26. Juli 1923.}

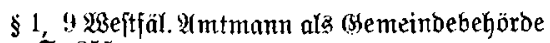
ธ. 255 .

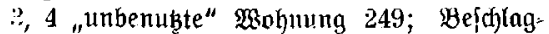
nahme, ohne zum Bertraṇid)luje Bohnumg. judyente zil bezeidnell 254.

4 3wangsmicte erit baut, went wenigitens zivei \$ohnumgiuchende bezeidhnet norbert waren 251; in Serlin geniigt bie Buweijung luar eines $342 ;$ Bebeutung bes $\$ 4^{1}$ a. F. 258 .

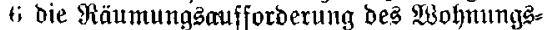

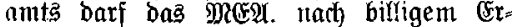
meffen aufbeben 255; Ynoronung einer

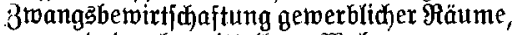
wenn baburd mittelbar Bohnuaum vere füųbar wito 344 ; hei ber Frage, ob eine Wohnung übergros ift, bleiben seubauten (\$12) unberiid(jid)tigt 345 .

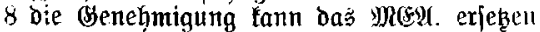
345.

9 Tie Anoronungen fint poweit jie sur 214 -

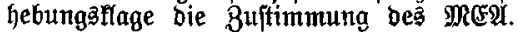
erforbern, jebt auser $\mathfrak{A r n f t}$ getreten 257 .

12 Gat ber BerfBeredigte bei ben Reubauten aus eigenen Mitteln neue æin̈tme gefdaffen, jo jallen biefe nicht unter bas $29 M(s)$. 252.

\section{Pr. Gejeks iiber ben Bexfehr mit Grund: ittiden}

v. 10. Fetruar 1993.

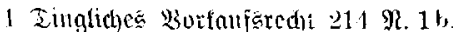

1 \$r. $2 a$ Sinholung boil 2 (usfünten 213 ; "genubtes ober nubbare

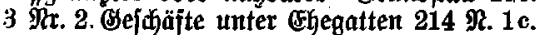

3 Nx. 4 Bentigt bie Benehmigung ?ur Barzel= lietung? $217 \Re .1$.

7 Beginn Der Frịt 214 u. $\mathfrak{N}$. 1d; Keringung zurzeit 214.

11 Frift fur bie Bejarverbcentifneioung 215 ; menn biefe fich auf bie Yufhebung ber $\mathfrak{B e r}=$ jagung befchränưt 215 .

15 (sefdäfte bor ber Eintraanug ber Smbs. 213 N. 1.

\section{Bibilprozejorbmung.}

3 bei nidjt beftimmter Mietbauer $121 \Re .2$; Räumung wegen nidhtigelt Saufs 122; nach

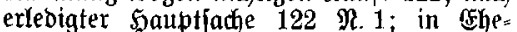

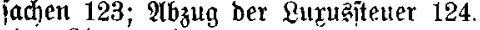

4 ober Ilagerweiterung 124.

29 affrebitierung 124/5

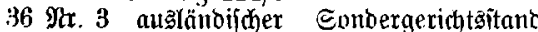

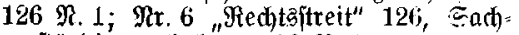
berftänbigengeblihren 126 凡. 1 .

40 Bereinbarung 25.

41 Sefelrfanfter bei Streitigfeiten ber Gmbs.

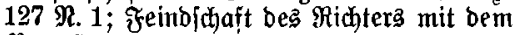
Prozepbertreter $127 \mathfrak{R} .1$

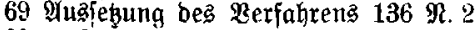

89 ertipreitende $\mathfrak{a n m e n d u t g} 127 \mathfrak{9} .2$.

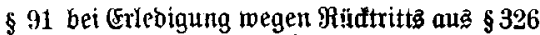
BGB. S. 128; für Sidjerheitşinterlegung 129; 'Säbrungş haben an ber Sidjerhet

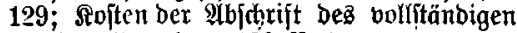
Ilrteils, Sutacftett $129 \Re .1$.

93 Sofortiges Unerł́enntniz 130.

99 Bebeutung beg $3 . \mathfrak{⿰} \mathfrak{b} \hat{j} .128$.

103 Fejtienung ber foften bes Sdfiebs. verfahrens 132.

104 Der Beridht

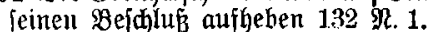

110 begenfeitigfeit 133.

115 भnfprüche bes $\mathfrak{Y}$ rmenanmalts gegen leine Parfei 135; Des dazu beftellten \$flegers 135

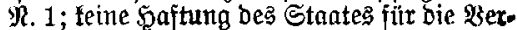
gütung bes Sequefters 167.

124 Gebühren des armen: und bes jpäter gemählten Jumalts 136.

253 Berhantolung ohne Flagezuftellung 136 Möngel biejer 137 .

271 sioitenenticheioung nad Sergleid 136.

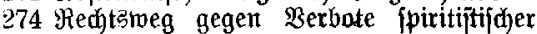
Sikungen 138; Enteignungen aus bem

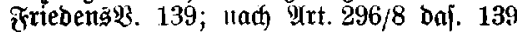
u. $\mathfrak{\Re} .3$.

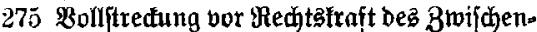
urteís 140.

291 Difenfunoigkeit burd) Beitunģberichte

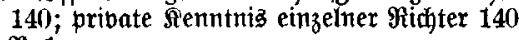
N. 1

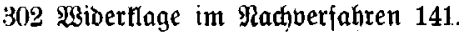

323 Sajtung bes erfackflichtigen Dritten 171; (Erhöhung des linterhalte 363 .

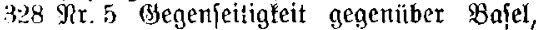

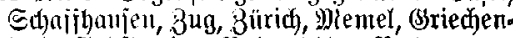
Inno, Süblatwien, ßolen 141 u. $\mathfrak{R} .1$; gegen: über ber Sđdmeiz 172.

344 Bebeutung 128.

356 Qäuterung fei Nichtzahlung des Bor \{dhuffes 145.

372 Drtstermine $144 \mathfrak{N} .1 \mathrm{~d}$

383 Bebeutung be Şaager 9bf. v. 17. 7. 05 भrt. $11^{3} 143$.

385 Zeugniäweigerung des Bantiers $143 \mathfrak{R} .1$; Witherruf ber Entbintung des $2 \mathfrak{r}_{z}$ tes bon jeiner Sdyweigepflidyt $143 \mathfrak{R}$. $1 \mathrm{c}$.

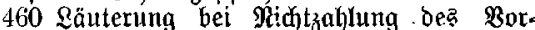
(d) uffes alı $\$ 356$ RßO. 145.

$465 / 7$ Yuzlegung 145 .

486 श(blehnung ber Einftellung 146.

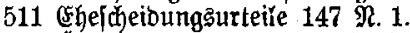

516 libergabe ber Berufungsidrift augerbalb des Dienjtgebäubes 147; an (Beridtsjăreiber anorer Senate $147 \Re .2$.

519 makgebeno ift ber in ber Berhandung gefterlte intrag $147 \mathfrak{R} .1$.

520 in Arreftjactien 147; 9 Nnfechtung ber $\mathfrak{B e}$.

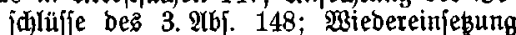
bei ber|äumter Bahlung 148 น. $\Re .1$.

536 bei Teilurteilen $43,149$.

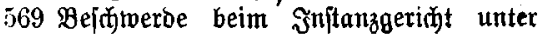
farjcher Unichrift 150.

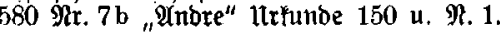

592 ff. Rotaubleşungen 150. 


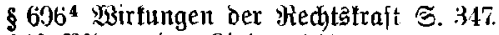

646 ßíleger ber ginoer 151.

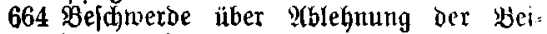
oronung eines Ynwalts:; Entziebung bes Urmented te 151 ). 1.

$712 / 3$ übergehung $152 \mathfrak{R} .1$; Burüđuahme ถes Redtafraftzeugnifjes 152 凡. 1.

739 bei (sinttertremung 152.

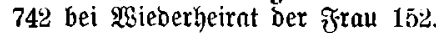

767 bei einjtro. Berīigungen 153; $\mathfrak{A} \mathfrak{i}=$ redfnung gegen die fiojtenfeftjesung 153

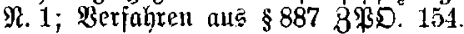

769 शuझ̆onoerunģflagen 154 .

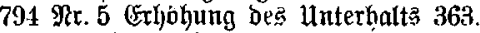

804 ßfänoung eigener sađhen 155.

821 Reihhaushtheine 155.

825 forbert gutgläubigen (snoerb 157

830 Fiändung einer im (Semahrjam eines Dritten befinolidien Briefgrunbichuld 157.

849 Bebeutung bes 9rnerfenttniffes 158.

848 cinuragung bes Sequefter 159; Eidhe rungshypothel 160 R. 1 .

866 wertbeftänoige 3 wangahnpothełen 161 ; megen Reichasteuern 234; aus Arrejturteilen 236 .

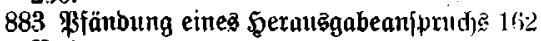
গ. 1 .

888 serausgabe bes snpobriejes 16:; (5r zmingung einer im sthlande borzunehmen= ben Salnolung 163; ber Utnderung eines Bereingnamens 163; úbertragung einer Ramenabatie 310.

894 bei einftro. Berfügungen 164; Sflnoerung eines Rereinģnamens 163.

895 Bufdhlag 155; Bolftredung nad) $\$ 887$

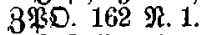

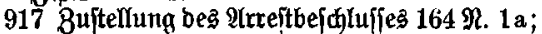
fiopten bei crlebigurtg bes Irreites 164 $\Re .1 \mathrm{~b}$.

924 Bebeutung 128.

926 wenn bie Urreftiorberung ingwifhen $\mathrm{im}$

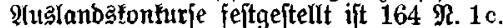

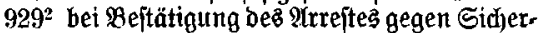
heit 165; bei Îrrejten ber Finamzämter 164 গ. $1 \mathrm{~d}$.

938 Senueftrntion eines \$anbelsgeiduñf! 166.

940 Berbote aus (Srunbbuchamt, ben Cipen tumämed fjel einzutragell 164, 165.

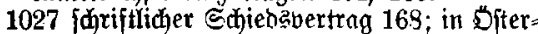
reidi) 168.

1034 Tragtweite einer Turdarbitrage 170.

1041 nidhtiger 3ertrag 169 II. N. 1.

1042 Olufred)nung gegen bie Olnge 171; 3urifthaltung 105 .

1045 Rergütung ber Sđjtebstidhter 169 R. 1 .

\section{Srunbbutjoronung.}

13 Crmittlungen bon $\mathfrak{A m}$ tämegen 173.

16 "Borbehalt" ber Afupertung 174; $B$ e belltung bes $2 . \mathfrak{Y b j}$. 176.

17 Behandlung 1 ; wenit alle 3wifdhenertwerber ihre (Eintragung gefonbett beantragen 177.

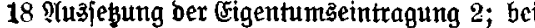
Betwilligung Ridhtberedtigter 178; beifeblen=
Der Steuerquittung S. $178 \mathfrak{R} .1$; \&ojhyung

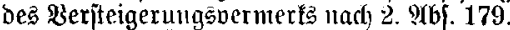

$\$ 19$ Finfeitige fillocumg $5.17 \%$

tot hei ßargellenbermedffung 180; inl ;ralle DQ $\$ 412$ R2SD. 180

28 \%ülegung des 1. Snkes 181/2; Heal!afteu, sonpotijeten in Bolomarł 11, 13; in Menten marf 182.

-9) 2lufbebung ber Benilligung 183; bei Prozeñuergleith $183 \mathfrak{N} .1$.

32 Berentigung zux Burümıhnte 176 .

36 11nzweibentiger (Erbfd)ein 185.

37 Fnhalt bes Beugniffes 1959 . 1.

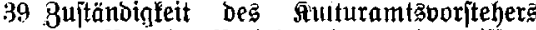

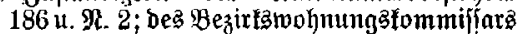

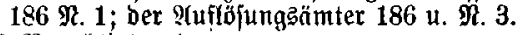

40 Beredigter 1.

41 bei fortnejegter (3). 18\%; bei qbtretung ber bum visuerben bezahiten supothet 190 .

48 bie Erflärung ber (Erwerber genügt 182; wenn dem Sohne unb beffen tunftiger Frau aujgelaffen ift $182 \mathfrak{N} .1$.

50 artenteil 193.

55 Befanntmaduung 194 $\mathfrak{R} .1$.

62 bei Binsänoerung 14

67 Srlebigung bes \& Intrags 177.

71 "Eintragung" 194.

80 Qanbesbant \$eftfalens 195; Sd)lesmig. Soljteins 195.

89 Madtragung ber Tatniterbezeidnung ber Wuenparzellen 195.

\section{3. (Sejeb ïber dic Angelegenteiten ber iretwilligen Geridgtaburteit.}

6) $\mathfrak{i x .} 4$,Beteiligter 196.

12 bei vorläufiger $\Re$ egelung $197 \mathfrak{R} 1$.

16 $\mathfrak{1 6 j . 1}$ gilt für bie (Ermäd)tgung zur $\mathfrak{B e}$. rujung einer Bereinsberjammlung 197.

20 Befdiwerbe des Bertrag̉sgegners 198; Des

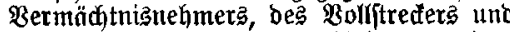
bes Grben 198 R. 1; Rblehnung einer Softenentfdyeibung bei crrlebigung bes $\mathfrak{A n}$ * trags $208 \Re$. 1 a.

29 zu Protolorl des Richter' 198; nur wegent Der Foîten 109.

34 Borlejen des 2ltteningalts 200 .

43 शttobehnung bes 2. 9(bi. 200; aui bie Schubaufficht 385.

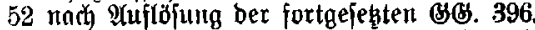

57 Nr. 9 Befanerbe bez Baters, ber fich mit bem Ginde vergleidhen will 366 .

59 mach Mitteilung ber Senehmigung 384 $\mathfrak{R} .1$.

60 פr. 1 gilt auch für bie $\mathfrak{B}$ efdntwerde Dritter 377.

126 Grmittlungspflicht bes Berid)ts, reenn bie

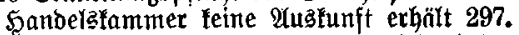
133 bie $\mathfrak{A}$ uflage bes $\$ 132$ ijt $\jmath^{u}$ wiederholen $197 \Re .2$

134 Beripäteter (Simiprudi 196.

142 Söjchuth des nur bom Erwerber an gemelbeten (Bejđäftşübergang 202 ; bes in ber Gntwidfung beftitblidien Betriebs 203; 


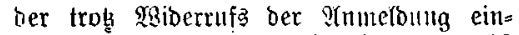
gctrngenen Bntk. S. 204 ; Des nus $\$ 29$

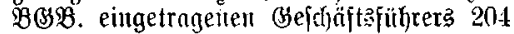

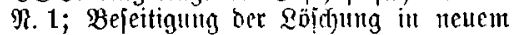
Berfahren 208.

\$ 144 Bebeutung ber $9(6 j .1,2205$ গ 1.

146 "Sören" 205; Bejđtwerbe ilber sill. tragung ver Riquibation 291.

147 Beifhmerde Des Rebifiontsbertallos 323.

159 Bernffung nad) \$ 37 BSB 6 . 197: \$rïfung, ob ber wirflidie. Borịtand den Serein an= gemeldet hat 206 .

163 Borallsfebungen $207 \Re$. 1 .

164 Bebelitung wer Wertsfeftiteflung 207.

169 , went die Brinbheit erft ant Ediluffe ber Berfanduntg jeftgeftellt wiro $208 \mathfrak{\Re} 1$.

193 Rart) Br(bef. v. 2. 1. 24 bexmittelt jebt bie Rotare Die ?hscinandarfebutg nus=

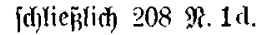

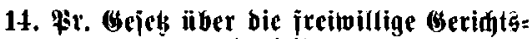 barfeit}

b. 21. Eeptember 1899.

Wrt. 1, rem jich bie Cuttidjeibung erlebigt fyat $\underline{208} \Re$. 1 b.

9, 11 હojortige meitere $\mathfrak{B}$ ejd)meroen mur wegen ber foften 199.

15 Feftipung ber Dromungajtraje fitr bie Sergatgenheit 208 .

32 Qbbfhriftsbeglatlinung un Falle beร $\$ 58$

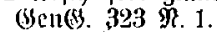

\section{Einzeluc Meidgatgeteke.}

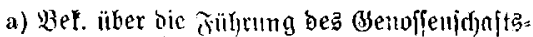
regititsrs 0 . 1. Yuli 1899

14 (sintragunts in nuageforichenter form 32? $\mathfrak{R} 1$.

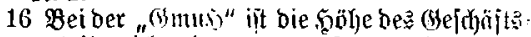
nolteils nidyt cillzutragen; fpäterc (5rhoshum $323 \Re .1 \mathrm{c}$. b) Sanger 96fommen itber ben Bibilprozeß̧ v. 17. ऊิuli 1905 .

\& 11 Bebeutung Des 3. Ykj. S. 143.

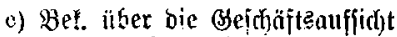
b. 14 . Dezember 1916.

$139 r .1$ Bergleidh 86 ; $9 x .2$ gegenüber bell 326 BSB. 37.

d) Friebenswertrag von Berjailles v. 28. Эuni 1919.

$276 \mathrm{c}, 277,289,291$ Gegenfeitigleit 133

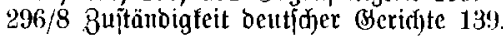

e) Reichboerfolfung v. 11. SYuguit 1919.

109 wirft nidht zurit 361.

114 Rerhtimeg 138.

153 Redutsmeg 139.

159 Bebeutung 70 গ. 2 b, 101.

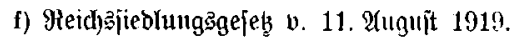
6 Borfaufąredt bei formmiorigent Bertrage 49. 11, 14 Iaujdjberträge 50 .

"r) Befeb ïber bie religiöfe (5rziehung ber Sinber v. 15. วuli 1921 .

1 Mif dheken 367.

2 Bebentung gegeniber ben $\$ 5368$.

5 FrElärung Des Sititoes 369.

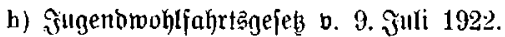

63 Mr. 2 Fürrjorgeerz̧iehung mintoerjäfriger UuĞänoer 371.

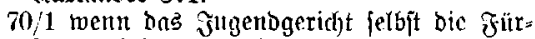
prgecrziehung anoronet 373 .

- i) Dritte @teuer= Motoeruromung . b. 14. Febr. 1924.

11 Burbegalt ber Fujhebung 174, 175; Qgieberciutrngung gelöphter soypothcfell 174 श. $1 \mathrm{c}$.

Earbuergedutis . . . . . . . . . . . . . . . . . . . . . . . . . 405

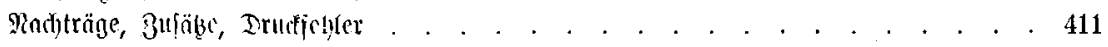


